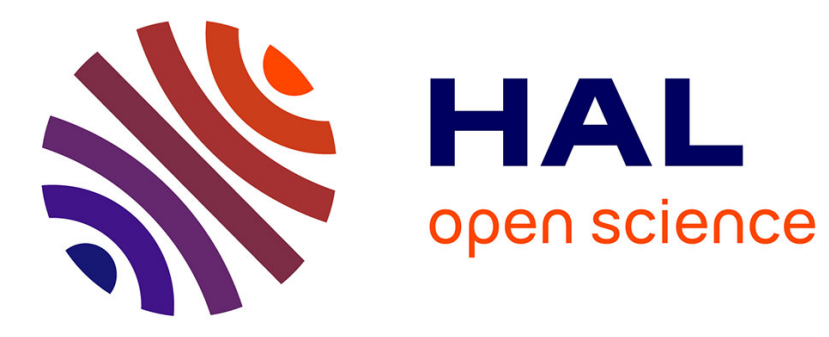

\title{
Twice: A Tilting Angle Amplification System for Parallel Robots
}

Sébastien Krut, Olivier Company, Frédéric Marquet, François Pierrot

\section{To cite this version:}

Sébastien Krut, Olivier Company, Frédéric Marquet, François Pierrot. Twice: A Tilting Angle Amplification System for Parallel Robots. ICRA: International Conference on Robotics and Automation, May 2002, Washington, United States. pp.4108-4113, 10.1109/ROBOT.2002.1014388 . lirmm00269414

\section{HAL Id: lirmm-00269414 https://hal-lirmm.ccsd.cnrs.fr/lirmm-00269414}

Submitted on 3 Apr 2008

HAL is a multi-disciplinary open access archive for the deposit and dissemination of scientific research documents, whether they are published or not. The documents may come from teaching and research institutions in France or abroad, or from public or private research centers.
L'archive ouverte pluridisciplinaire HAL, est destinée au dépôt et à la diffusion de documents scientifiques de niveau recherche, publiés ou non, émanant des établissements d'enseignement et de recherche français ou étrangers, des laboratoires publics ou privés. 


\title{
Twice: A Tilting Angle Amplification System for Parallel Robots
}

\author{
Sébastien Krut, Olivier Company, Frédéric Marquet, François Pierrot \\ LIRMM, UMR 5506, CNRS - Université Montpellier 2 \\ 161 rue Ada , 34392 Montpellier Cedex 5, France \\ $\{k r u t$, company, marquet, pierrot $\} @$ lirmm.fr
}

\begin{abstract}
Twice (Tilting device with WIde Capacity) a new amplification device able to double the tilting angle of a parallel mechanism is presented. A singularity analysis is carried out: it proves that Twice can give a huge tilting angle to Parallel Kinematic Machines (PKM). Finally, practical implementation features are discussed.
\end{abstract}

\section{Introduction}

After Gough and Stewart in the 50's and 60's introducing the idea of so-called "hexapods" [1][2], Clavel and his Delta structure [3] in the late 80's opened a new era of PKM able to reach extremely high velocities and accelerations. In addition, mechanism stiffness is often very high compared to serial arms. Thus many PKM have been dedicated to pick-and-place and more recently to machining.

But one of the PKM drawbacks is their limited workspace, especially the limitation of the nacelle's tilting angle. Four different ways may be possible to compensate for this drawback:

- Sharing the degrees-of-freedom (dof) between a carrying structure and a wrist, as it is done by $D S$ Technologies inc. with Sprint (serial + parallel), or Neos with Tricept [4] (parallel + serial). Note that the wrist could even be itself parallel, for example based on Agile Eye technology [5];

- Designing machines using the "right-hand / left-hand" paradigm, where both the tool and the manufactured part are moving with respect to the ground, as it is done in classical machines with turning tables;

- Building redundant machines, that is to say overactuated ones, like the Eclipse [6] machine tool or the Archi [7] robot. The Eclipse is designed to achieve five faces milling and Archi is a planar 3 degrees-of-freedom robot that allows an infinite rotation about one axis;

- Building mechanical amplification systems to increase tilting angles of existing machines. On the $\mathrm{H} 4$ robot [8], such a device exists: gears multiply by four the orientation angle, giving the tool a 360-degrees rotation capability. But a gear system can only be used to amplify one rotation. What is proposed here, is a mechanical device able to double tilting angles of the nacelle about two rotation axis. This alternative solution has clearly the advantage of its simplicity; upgrading an existing machine or implementing a new one is very simple. This mechanical device, with no additional actuator, allows high dynamics performances thanks to its parallel design.

In this paper, the Twice (Tilting device with WIde Capacity) amplification system is firstly introduced as an add-on device for parallel machine-tool or robot. Then a detailed internal velocities analysis is carried out to prove the mechanism efficiency, that is to say it does not suffer from singular positions within a huge workspace in rotation. Finally, some practical implementation examples of Twice are proposed.

\section{The Twice concept}

To introduce the Twice concept, let us consider a 5-dof PKM. Machine in Figure 1 is similar to Zamanov's architecture [9]. It is composed of 4 identical P-S-S chains (P stands for prismatic, $\mathrm{S}$ for spherical), plus a P-U-U chain (U means universal) giving 5 dof to the ending part.

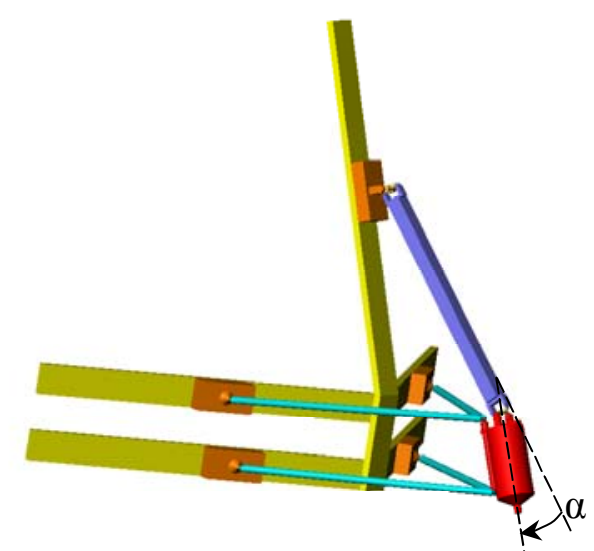

Figure 1 - 5-dof parallel machine-tool

To avoid singularities, i.e. to have a good transmission of velocities and forces, the tilting angle must remain low: that is to say $\alpha \leq 45^{\circ}$ on this machine. In most PKM, the tool (a spindle, a gripper, ...) is rigidly fixed on the nacelle, thus the limitations on nacelle tilting angles directly limit the tool capability. What is discussed in this paper is the possibility of adding an amplification device 
between the PKM nacelle and the tool, that doubles tilting angles (Figure 2).

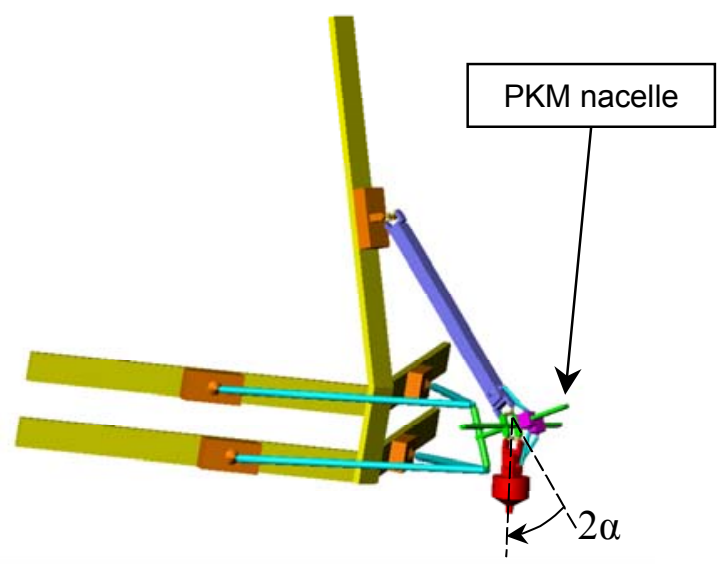

Figure 2 - 5 dof parallel machine tool with Twice

For that purpose, Twice is introduced in Figure 3: the nacelle is not rigid anymore, but a special mechanical device is inserted between the actuating arms and the tool. Considered from the middle part, this device presents a mirror symmetry: this is basically the reason why, when the nacelle PKM is tilted of a given angle, the tool is tilted of twice this angle.

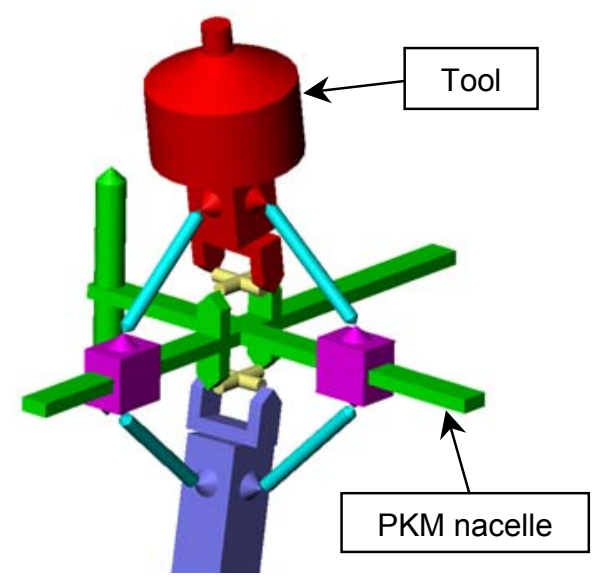

Figure 3 - CAD representation of Twice

The basic principle of Twice is shown in Figure 4 where each box stands for a joint. A first $U$ joint supports the mechanism and input rotational motions are intended to occur at this level. This is why this joint is in a grey box: would Twice be isolated from its PKM base, the two pivot joints of the U-joint should be actuated. Of course, when Twice is mounted on top of a PKM, these motions are the actual motions of the PKM nacelle.

If some geometrical constrains are fulfilled, the mobile platform is tilted by the same angles about the axis of the second universal joint. Both orientation actions will be added, providing the moving platform orientation with capabilities that are twice as large as the original ones, as long as no singularity occurs.

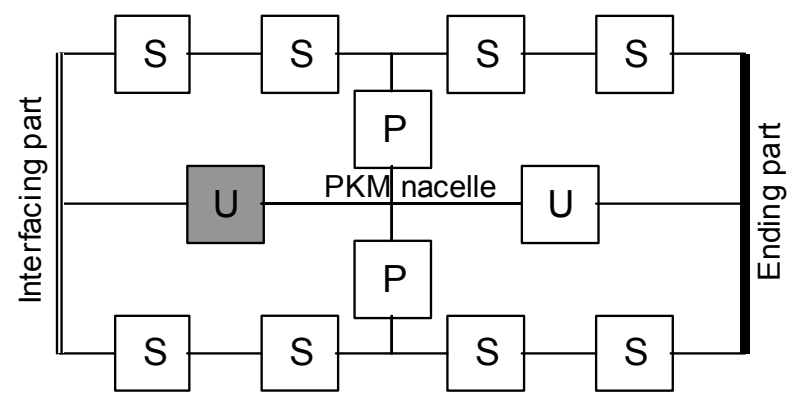

Figure 4 - Joints-and-Loops graph of Twice

Considering that the support PKM is not in a singular position (i.e. the Twice input motions are correctly addressed), the following analysis will only point out the internal singularities of the device.

\section{Kinematic model analysis}

In this part the validity of this device will be proved.

\subsection{Mobility analysis}

Referring to Figure 4, mechanism characteristics are: $n=12$ (number of joints),

$p=9$ (number of parts).

Owing to the duality between velocities and forces, it is equivalent to search singularities by studying internal forces or internal velocities.

$>$ Analysis of internal velocities

$v=n-p+1=4$, independent chains,

$E_{c}=6 v=24$, equations given by a kinematic study,

$I_{c}=2 \times 1+8 \times 3+2 \times 2=30$, unknown velocities.

$>$ Analysis of internal forces

$E_{S}=6(p-1)=48$, forces equations,

$I_{S}=2 \times 5+8 \times 3+2 \times 4=42$, forces unknowns.

The above statement means that a kinematic analysis (concerning internal velocities) will involve less equations and unknowns than a forces analysis. Thus the following analysis will be focused on internal velocities. Then, the mobility index is then given by:

$$
m=I_{c}-E_{c}=6 .
$$

Noticing that the four S-S bars can rotate about their axis, and assuming that the system is isostatic, the useful kinematic mobility index is equal to two, which indicates that if the system is not singular, this device has two degrees-of-freedom. 
To go further, forward kinematic model needs to be established.

\subsection{Kinematic model parameters}

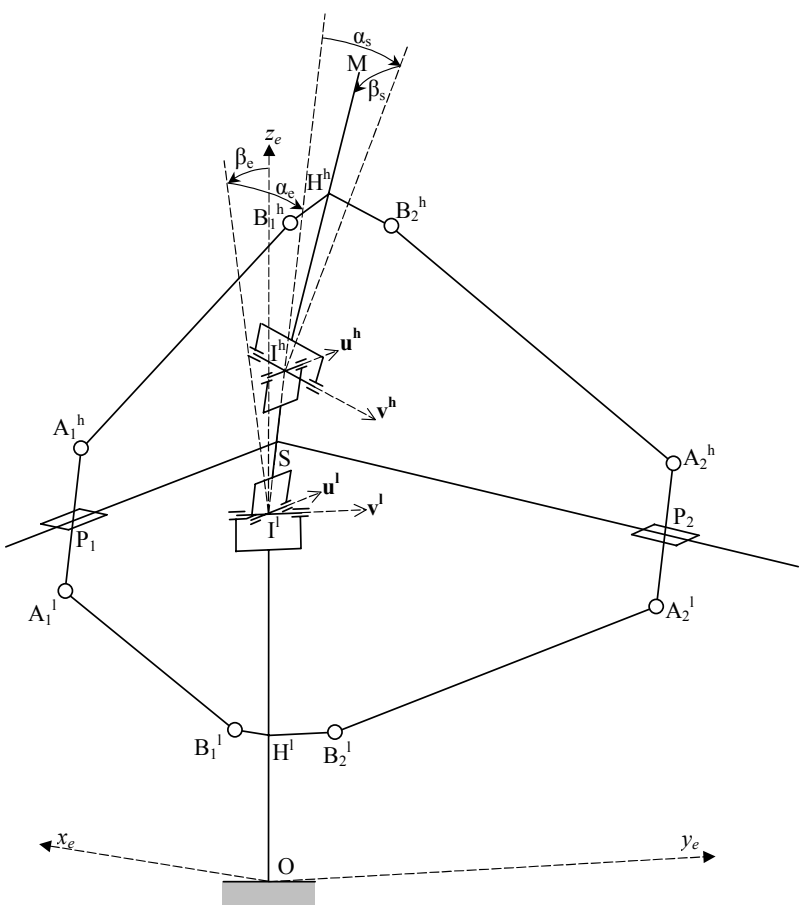

Figure 5 - Kinematic scheme of Twice

The geometrical parameters of Twice are:

$\left\|\mathbf{S} \mathbf{I}^{\mathbf{h}}\right\|=\left\|\mathbf{S I}^{\mathbf{I}}\right\|=a$, half distance between the centers of both universal joints,

$\left\|\mathbf{I}^{\mathbf{l}} \mathbf{H}^{\mathbf{l}}\right\|=\left\|\mathbf{I}^{\mathbf{h}} \mathbf{H}^{\mathbf{h}}\right\|=b$,

$\left\|\mathbf{O H}^{\mathbf{I}}\right\|=c^{l}$, where $\mathrm{O}$ represents the machine origin,

$\left\|\mathbf{H}^{\mathbf{h}} \mathbf{M}\right\|=c^{h}$, where $\mathbf{M}$ is the extremity of the ending part,

$\left\|\mathbf{P}_{\mathbf{1}} \mathbf{A}_{\mathbf{1}}^{\mathbf{l}}\right\|=\left\|\mathbf{P}_{\mathbf{1}} \mathbf{A}_{\mathbf{1}}^{\mathbf{h}}\right\|=\left\|\mathbf{P}_{\mathbf{2}} \mathbf{A}_{\mathbf{2}}^{\mathbf{l}}\right\|=\left\|\mathbf{P}_{\mathbf{2}} \mathbf{A}_{\mathbf{2}}^{\mathbf{h}}\right\|=e$,

$\left\|\mathbf{H}^{\mathbf{l}} \mathbf{B}_{\mathbf{1}}^{\mathbf{l}}\right\|=\left\|\mathbf{H}^{\mathbf{l}} \mathbf{B}_{\mathbf{2}}^{\mathbf{l}}\right\|=\left\|\mathbf{H}^{\mathbf{h}} \mathbf{B}_{\mathbf{1}}^{\mathbf{h}}\right\|=\left\|\mathbf{H}^{\mathbf{h}} \mathbf{B}_{\mathbf{2}}^{\mathbf{h}}\right\|=f$,

$\left\|\mathbf{A}_{\mathbf{1}}^{\mathrm{l}} \mathbf{B}_{\mathbf{1}}^{\mathbf{l}}\right\|=\left\|\mathbf{A}_{\mathbf{2}}^{\mathbf{l}} \mathbf{B}_{\mathbf{2}}^{\mathbf{l}}\right\|=\left\|\mathbf{A}_{\mathbf{1}}^{\mathbf{h}} \mathbf{B}_{\mathbf{1}}^{\mathbf{h}}\right\|=\left\|\mathbf{A}_{\mathbf{2}}^{\mathbf{h}} \mathbf{B}_{\mathbf{2}}^{\mathbf{h}}\right\|=l$, length of bars.

\section{(|| || stands for the cartesian norm)}

\subsection{Inverse kinematic model}

It must be pointed out that this device is symmetrical when considering the central part. Therefore it suffices to consider only Twice upper part shown in Figure 6: it is used to establish the forward and inverse kinematic models.

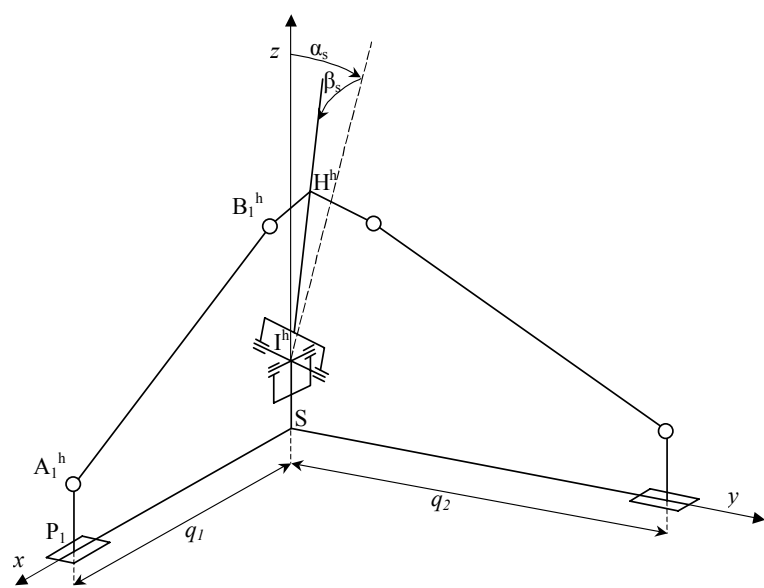

Figure 6 - Kinematic scheme of Twice upper part

In this Section the relationship between tool angles with respect to the central part, $\left(\alpha_{s}, \beta_{s}\right)$, and the position of the sliding parts, $\left(q_{1}, q_{2}\right)$, is derived. To do so, we expressed the fact that the length of $\mathbf{A}_{\mathbf{i}}^{\mathbf{h}} \mathbf{B}_{\mathbf{i}}^{\mathbf{h}}$, is known:

$$
\left\|\mathbf{A}_{\mathbf{i}}^{\mathbf{h}} \mathbf{B}_{\mathbf{i}}^{\mathbf{h}}\right\|^{2}=l^{2}, \quad \mathbf{i} \in\{1,2\},
$$

with $\mathbf{A}_{\mathbf{i}}^{\mathbf{h}} \mathbf{B}_{\mathbf{i}}^{\mathbf{h}}=\mathbf{P}_{\mathbf{i}} \mathbf{S}+\mathbf{A}_{\mathbf{i}}^{\mathbf{h}} \mathbf{P}_{\mathbf{i}}+\mathbf{S} \mathbf{I}^{\mathbf{h}}+\mathbf{I}^{\mathbf{h}} \mathbf{B}_{\mathbf{i}}^{\mathbf{h}}$, where:

$$
\mathbf{I}^{\mathbf{h}} \mathbf{B}_{\mathbf{1}}^{\mathbf{h}}=\operatorname{Rot}_{\alpha_{s}} \operatorname{Rot}_{\beta_{s}}\left[\begin{array}{l}
f \\
0 \\
b
\end{array}\right], \mathbf{I}^{\mathbf{h}} \mathbf{B}_{\mathbf{2}}^{\mathbf{h}}=\operatorname{Rot}_{\alpha_{s}} \operatorname{Rot}_{\beta_{s}}\left[\begin{array}{l}
0 \\
f \\
b
\end{array}\right],
$$

$\operatorname{Rot}_{\alpha_{s}}=\left[\begin{array}{ccc}1 & 0 & 0 \\ 0 & \cos \left(\alpha_{s}\right) & \sin \left(\alpha_{s}\right) \\ 0 & -\sin \left(\alpha_{s}\right) & \cos \left(\alpha_{s}\right)\end{array}\right], \operatorname{Rot}_{\beta_{s}}=\left[\begin{array}{ccc}\cos \left(\beta_{s}\right) & 0 & \sin \left(\beta_{s}\right) \\ 0 & 1 & 0 \\ -\sin \left(\beta_{s}\right) & 0 & \cos \left(\beta_{s}\right)\end{array}\right]$.

Let us note:

$q_{i}=\left\|\mathbf{P}_{\mathbf{i}} \mathbf{S}\right\|, \mathbf{U}_{1}=-q_{1} \mathbf{x}, \mathbf{U}_{\mathbf{2}}=-q_{2} \mathbf{y}, \mathbf{V}_{\mathbf{i}}=\left[\begin{array}{c}0 \\ 0 \\ a-e\end{array}\right]+\mathbf{I}^{\mathbf{h}} \mathbf{B}_{\mathbf{i}}^{\mathbf{h}}$, then (2) can be written as:

$$
\left\|\mathbf{A}_{\mathbf{i}}^{\mathbf{h}} \mathbf{B}_{\mathbf{i}}^{\mathbf{h}}\right\|^{2}=\left\|\mathbf{U}_{\mathbf{i}}\right\|^{2}+2 \mathbf{U}_{\mathbf{i}}{ }^{t} \cdot \mathbf{V}_{\mathbf{i}}+\left\|\mathbf{V}_{\mathbf{i}}\right\|^{2} .
$$

This leads to second order polynomial equations:

$$
\begin{gathered}
q_{1}^{2}+2 q_{1}\left(-\mathbf{x}^{t} \cdot \mathbf{V}_{\mathbf{1}}\right)+\left\|\mathbf{V}_{\mathbf{1}}\right\|^{2}-l^{2}=0, \\
q_{2}{ }^{2}+2 q_{2}\left(-\mathbf{y}^{t} \cdot \mathbf{V}_{\mathbf{2}}\right)+\left\|\mathbf{V}_{\mathbf{2}}\right\|^{2}-l^{2}=0 .
\end{gathered}
$$

According to the choice to count $q_{1}\left(q_{2}\right)$ negative in the direction of vector $\boldsymbol{x}$ (respectively $\boldsymbol{y}$ ), only the largest root of the polynomial is considered since it corresponds to the proper configuration of the mechanism.

Thus a unique relationship can be established between the tool orientation (with respect to Twice central part) 
and the positions of the two sliding parts. Due to the symmetrical design of Twice, the same relationship exists between the orientation of the central part (with respect to Twice base) and the position of the very same sliding parts. This proves that:

$$
\begin{aligned}
& \alpha_{s}=\alpha_{e}=\alpha, \\
& \beta_{s}=\beta_{e}=\beta .
\end{aligned}
$$

So, if $\mathfrak{R}_{e}$ is the frame attached to the Twice base, $\mathfrak{R}$ the one attached to the central part and $\mathfrak{R}_{s}$ the one attached to the tool, Figure 7 expresses the changes between $\mathfrak{R}_{e}, \mathfrak{R}$ and $\mathfrak{R}_{s}$ :

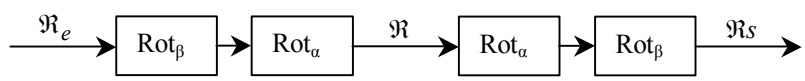

Figure 7 - Frame transformation

Consequently, the tool orientation with respect to the base is twice the orientation of the central part (as long as no singularity occurs).

\subsection{Singularity analysis}

For most parallel mechanisms, a simple analysis of the input-output velocity relationship is sufficient to carry out a singularity analysis. Here a more detailed study is proposed, which enlightens the full expression of all joints velocities (internal velocities). Indeed, if the matrix mapping input velocities to internal velocities can be inverted, the mechanism is not singular. Of course, the computation of this matrix will be done only once during the machine design process.

The four loops closure equations have to be expressed to compute internal velocities. As an example, let us consider one loop of the upper part (upper right loop in Figure 6). This leads to:

$$
\left\{\begin{array}{c}
\mathbf{V}_{O h / O p}^{\mathrm{I}^{\mathrm{h}}}+\mathbf{V}_{2 h / O h}^{\mathrm{I}^{\mathrm{h}}}+\mathbf{V}_{2 p / 2 h}^{\mathrm{I}^{\mathrm{h}}}+\mathbf{V}_{O p / 2 p}^{\mathrm{I}^{\mathrm{h}}}=\mathbf{0} \\
\boldsymbol{\Omega}_{O h / O p}+\boldsymbol{\Omega}_{2 h / O h}+\boldsymbol{\Omega}_{2 p / 2 h}+\boldsymbol{\Omega}_{O p / 2 p}=\mathbf{0}
\end{array}\right.
$$

where $O h$ is the ending part, $2 h$ is the upper right bar, $2 p$ is the right master part of the prismatic joint and $0 p$ is the middle part. For instance vector $\mathbf{V}_{O h / O p}^{\mathrm{I}^{\mathrm{h}}}$ represents the velocity of part $O h$ seen from $O p$ at point $\mathrm{I}^{\mathrm{h}}$.

Velocities of each joints are:

$$
\left\{\begin{array}{c}
\mathbf{V}_{O p / 2 p}^{\mathrm{P}_{2}}=-V_{P_{2}} \cdot \mathbf{y} \\
\mathbf{\Omega}_{O p / 2 p}=\mathbf{0}
\end{array}\right.
$$

$$
\begin{gathered}
\left\{\begin{array}{c}
\mathbf{V}_{0 h / 0 p}^{\mathrm{I}^{\mathrm{h}}}=\mathbf{0} \\
\mathbf{\Omega}_{O h / 0 p}=\dot{\alpha}_{s} \mathbf{u}_{\mathbf{h}}+\dot{\beta}_{s} \mathbf{v}_{\mathbf{h}}
\end{array}\right. \\
\left\{\begin{array}{c}
\mathbf{V}_{2 h / 0 p}^{\mathrm{B}_{2}^{\mathrm{h}}}=\mathbf{0} \\
\mathbf{\Omega}_{2 h / 0 h}=\omega_{2 h / 0 h}^{x} \mathbf{x}+\omega_{2 h / 0 h}^{y} \mathbf{y}+\omega_{2 h / 0 h}^{z} \mathbf{z}
\end{array}\right. \\
\left\{\begin{array}{c}
\mathbf{V}_{2 p / 2 h}^{\mathrm{A}}=\mathbf{0} \\
\mathbf{\Omega}_{2 p / 2 h}^{\mathrm{h}}=\omega_{2 p / 2 h}^{x} \mathbf{x}+\omega_{2 p / 2 h}^{y} \mathbf{y}+\omega_{2 p / 2 h}^{z} \mathbf{z}
\end{array}\right.
\end{gathered}
$$

where $\mathbf{x}, \mathbf{y}, \mathbf{z}$ are frame $\mathfrak{R}$ base vectors. For now on, all vectors will be expressed by their coordinates in $\mathfrak{R}$ :

$\mathbf{u}^{\mathbf{h}}=\left[\begin{array}{c}-1 \\ 0 \\ 0\end{array}\right], \mathbf{v}^{\mathbf{h}}=\operatorname{Rot}_{\boldsymbol{\alpha}}\left[\begin{array}{l}0 \\ 1 \\ 0\end{array}\right]$.

Equation (8) leads to:

$$
\mathbf{I}^{\mathbf{h}} \mathbf{B}_{\mathbf{2}}^{\mathbf{h}} \times \boldsymbol{\Omega}_{2 h / 0 h}+\mathbf{I}^{\mathbf{h}} \mathbf{A}_{\mathbf{2}}^{\mathbf{h}} \times \boldsymbol{\Omega}_{2 p / 2 h}+\mathbf{V}_{0 p / 2 p}^{\mathrm{P} 2}=\mathbf{0},
$$

( $\times$ denotes the cross product) with:

$$
\begin{aligned}
& \mathbf{I}^{\mathbf{h}} \mathbf{B}_{\mathbf{2}}^{\mathbf{h}}=\operatorname{Rot}_{\alpha} \operatorname{Rot}_{\beta}\left[\begin{array}{l}
0 \\
f \\
b
\end{array}\right], \mathbf{I}^{\mathbf{h}} \mathbf{A}_{\mathbf{2}}^{\mathbf{h}}=\operatorname{Rot}_{\alpha} \operatorname{Rot}_{\beta}\left[\begin{array}{c}
0 \\
q_{2} \\
e-a
\end{array}\right] \text { and } \\
& \mathbf{B}_{\mathbf{2}}^{\mathbf{h}} \mathbf{A}_{\mathbf{2}}^{\mathbf{h}}=\mathbf{I}^{\mathbf{h}} \mathbf{A}_{\mathbf{2}}^{\mathbf{h}}-\mathbf{I}^{\mathbf{h}} \mathbf{B}_{2}^{\mathbf{h}} .
\end{aligned}
$$

To be able to solve this system, the internal angular velocity of the upper right bar about its own axis must be set, for example set to zero:

$$
\mathbf{\Omega}_{2 h / 0 h} \cdot \mathbf{B}_{\mathbf{2}}^{\mathbf{h}} \mathbf{A}_{\mathbf{2}}^{\mathbf{h}}=0 .
$$

Finally the loop closure is expressed by:

$$
\mathbf{M}_{\mathbf{2 h}}\left[\begin{array}{lllllllll}
\dot{\alpha}_{s} & \dot{\beta}_{S} & V_{P 2} & \omega_{2 h / 0 h}^{x} & \omega_{2 h / 0 h}^{y} & \omega_{2 h / 0 h}^{z} & \omega_{2 p / 2 h}^{x} & \omega_{2 p / 2 h}^{y} & \omega_{2 p / 2 h}^{z}
\end{array}\right]^{t}=\mathbf{0},
$$

where $\mathrm{M}_{2 \mathrm{~h}}$ is a $7 \times 9$ matrix:

$$
\begin{aligned}
& \mathbf{M}_{\mathbf{2 h}}= \\
& {\left[\begin{array}{cc:c:ccc:ccc}
u_{x}^{h} & v_{x}^{h} & 0 & 1 & 0 & 0 & 1 & 0 & 0 \\
u_{y}^{h} & v_{y}^{h} & 0 & 0 & 1 & 0 & 0 & 1 & 0 \\
u_{z}^{h} & v_{z}^{h} & 0 & 0 & 0 & 1 & 0 & 0 & 1 \\
\hdashline 0 & 0 & 0 & B_{2}^{h} A_{2}^{h x} & B_{2}^{h} A_{2}^{h y} & B_{2}^{h} A_{2}^{h z} & 0 & 0 & 0 \\
\hdashline 0 & 0 & 0 & 0 & -I^{h} B_{2}^{h z} & I^{h} B_{2}^{h y} & 0 & -I^{h} A_{2}^{h z} & I^{h} A_{2}^{h y} \\
0 & 0 & -1 & I^{h} B_{2}^{h z} & 0 & -I^{h} B_{2}^{h x} & I_{2}^{h} A_{2}^{h z} & 0 & -I^{h} A_{2}^{h x} \\
0 & 0 & 0 & -I^{h} B_{2}^{h x} & I^{h} B_{2}^{h x} & 0 & -I_{2}^{h} A_{2}^{h y} & I_{2}^{h} A_{2}^{h x} & 0
\end{array}\right]}
\end{aligned}
$$

First 3 lines of matrix $\mathbf{M}_{\mathbf{2 h}}$ correspond to equation (9), line 4 is equation (15) and the last lines correspond to equation (14). 
Same calculus can be done for the three other chains, leading to three $7 \times 9$ matrices, denoted $\mathbf{M}_{1 \mathbf{h}}, \mathbf{M}_{21}, \mathbf{M}_{11}$. The global relationship system is given by:

$$
\mathbf{M}_{\mathbf{t}} \mathbf{V}_{\mathbf{i}}=\mathbf{V}_{\mathbf{0}} \text {, }
$$

where:

$$
\mathbf{M}_{\mathbf{t}}=\left[\begin{array}{cccc}
\mathbf{M}_{\mathbf{2 h}} & 0 & 0 & 0 \\
0 & \mathbf{M}_{1 \mathbf{h}} & 0 & 0 \\
0 & 0 & \mathbf{M}_{2 \mathbf{l}} & 0 \\
0 & 0 & 0 & \mathbf{M}_{1 \mathbf{l}} \\
\hdashline & \mathbf{M}_{\mathbf{b}} &
\end{array}\right] .
$$

$\mathbf{V}_{\mathrm{i}}$ is a $36 \times 1$ vector containing all internal velocities: $\mathbf{V}_{\mathbf{i}}^{t}=$

$$
\left[\begin{array}{lllllllll}
\dot{\alpha}_{s} & \dot{\beta}_{s} & V_{P 2} & \omega_{2 h / 0 h}^{x} & \omega_{2 h / 0 h}^{y} & \omega_{2 h / 0 h}^{z} & \omega_{2 p / 2 h}^{x} & \omega_{2 p / 2 h}^{y} & \omega_{2 p / 2 h}^{z} \\
\dot{\alpha}_{s} & \dot{\beta}_{s} & V_{P 1} & \omega_{1 h / 0 h}^{x} & \omega_{1 h / 0 h}^{y} & \omega_{1 h / 0 h}^{z} & \omega_{1 p / 1 h}^{x} & \omega_{1 p / 1 h}^{y} & \omega_{1 p / 1 h}^{z} \\
\dot{\alpha}_{e} & \dot{\beta}_{e} & V_{P 2} & \omega_{2 l / 0 l}^{x} & \omega_{2 l / 0 l}^{y} & \omega_{2 l / 0 l}^{z} & \omega_{2 p / 2 l}^{x} & \omega_{2 p / 2 l}^{y} & \omega_{2 p / 2 l}^{z} \\
\dot{\alpha}_{e} & \dot{\beta}_{e} & V_{P 1} & \omega_{1 / / 0 l}^{x} & \omega_{1 / / 0 l}^{y} & \omega_{1 / / 0 l}^{z} & \omega_{1 p / 1 l}^{x} & \omega_{1 p / 1 l}^{y} & \omega_{1 p / 1 l}^{z}
\end{array}\right]
$$

$\mathbf{V}_{\mathbf{0}}$ is a $36 \times 1$ vector containing input velocities:

$\mathbf{V}_{\mathbf{o}}{ }^{t}=\left[\begin{array}{lllll}0 & \cdots & 0 & \dot{\alpha}_{e} & \dot{\beta}_{e}\end{array}\right]$,

$\mathbf{M}_{\mathbf{b}}$ is a $8 \times 36$ matrix. Due to lack of space, the expression of $\mathbf{M}_{\mathbf{b}}$ is not detailed here. We simply give its features:

- Its 6 first lines express the fact that some internal velocities appear two times in the internal velocity vector $\mathbf{V}_{\mathbf{i}}$. This concerns $\dot{\alpha}_{s}, \dot{\beta}_{s}, \dot{\alpha}_{e}, \dot{\beta}_{e}, V_{P 1}$ and $V_{P 2}$. For example, first line of this matrix regards $\dot{\alpha}_{s}$ : line is full of zero except at columns number 1 and 10 whose elements are respectively 1 and -1 .

- The two last lines of this matrix contain the input velocities; they are full of zero except at the $28^{\text {th }}$ column for the first and at the $29^{\text {th }}$ column for the last line: they link $\dot{\alpha}_{e}$ and $\dot{\beta}_{e}$ contained in vector $\mathbf{V}_{\mathbf{i}}$ with those contained in $\mathbf{V}_{\mathbf{0}}$.

The result of this calculus is the $36 \times 36$ matrix $\mathbf{M}_{\mathbf{t}}$ corresponding to the linear system linking internal velocities to command ones. It is a square matrix, which is normal because this mechanism is isostatic and that the rotation of bars about their own axis are taken into account.

If this system can be inverted, all internal velocities can be solved and the system is not singular. One way of verifying this point is to calculate the determinant of this matrix and to check that it never goes to zero.

\subsection{Case study}

Key dimensions (Figure 5) are: $a=10, b=30, e=10, f=10$ and $l=50$.
Figure 8 shows the value of the determinant of $\mathbf{M}_{\mathbf{t}}$ for a tilting angle of the nacelle lower than $45^{\circ}$ (the tool can be oriented in a complete hemisphere). This involves that $\alpha$ and $\beta$ must verify the relation:

$$
\cos (\alpha) \times \cos (\beta) \geq \cos \left(45^{\circ}\right)
$$

In this plot, the maximal value of the determinant is $3.1 \mathrm{e}+023$, the lowest value is $1.5 \mathrm{e}+022$. This represents a ratio of 20.4. In the whole range of $\alpha$ and $\beta$ the determinant remains positive. It means that the system leading to internal velocities can always be inverted, in other words, that the mechanism is never singular.

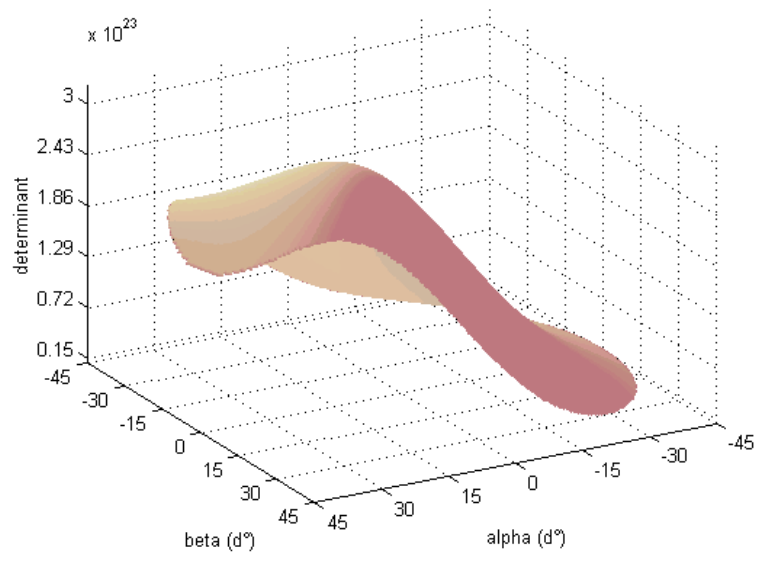

Figure 8 - Relative variations of the determinant

Figure 9 represents this mechanism for $\alpha$ starting at 0 going to $30^{\circ}$ and $\beta$ starting at 0 going to $60^{\circ}$. As can be seen, a tilting angle of $45^{\circ}$ or more, can be reached.

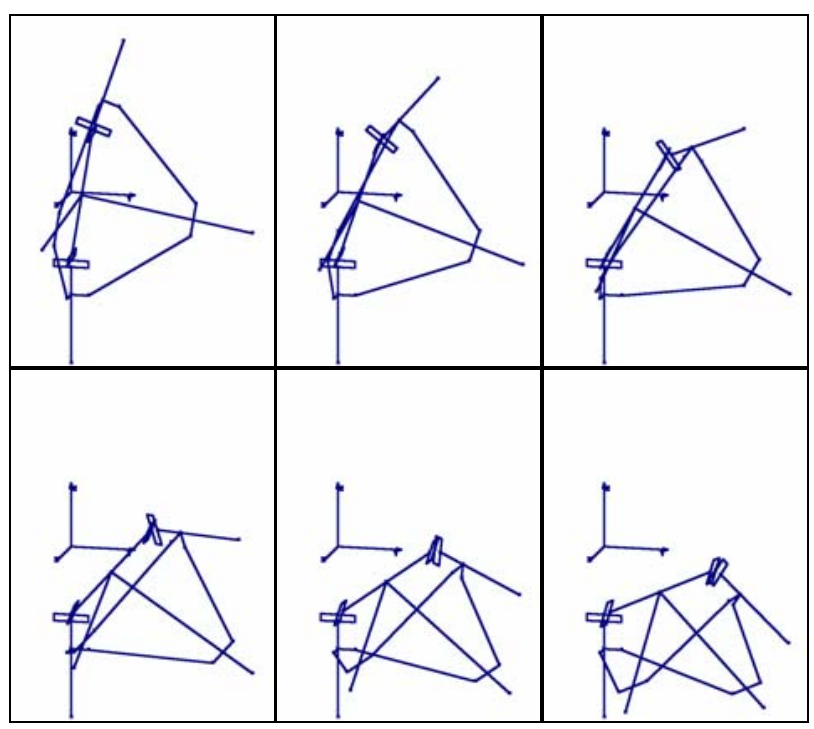

Figure 9 - CAD representation of a Twice motion 


\section{Practical implementations}

Twice basic mechanism can be improved by adding redundant legs. The mechanism becomes overconstrained, but internal forces are balanced, and the mechanism is viable. Figure 10 shows a version of Twice with four sliding parts, and Figure 11 shows a version with three sliding parts. Since no prototype was built, problems regarding accuracy and stiffness are still to be studied.
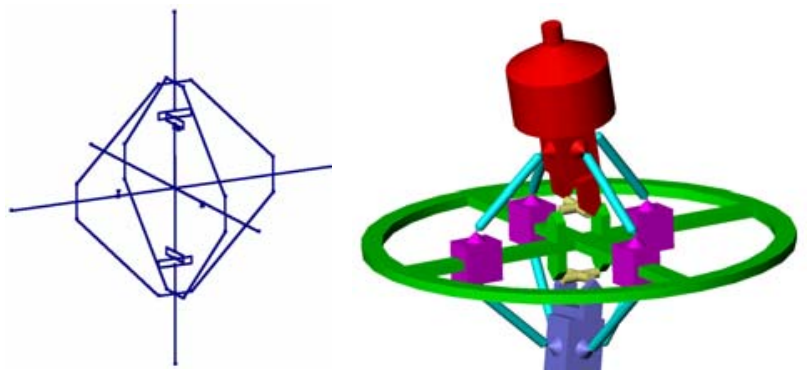

Figure 10 - Twice with four prismatic joints
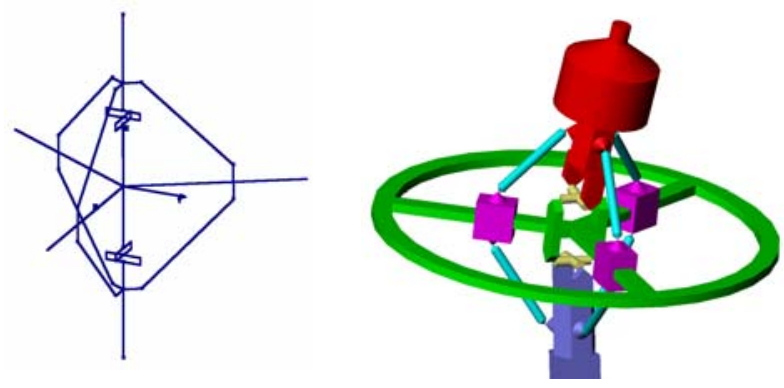

Figure 11 - Twice with three prismatic joints

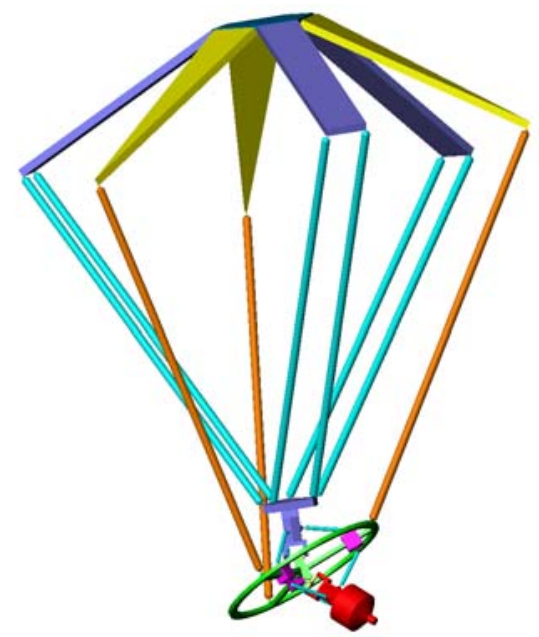

Figure 12 - Delta with Twice-wrist

On the other hand, Twice mechanism can be mounted on various carriers, including a Delta robot. In such a case, the Twice inputs may be arranged as in Figure 12: R-S-S actuated chains are connected to Twice central part, while the first Twice U-Joint is mounted on the Delta's nacelle. In the presented arrangement, actuation redundancy is envisioned for the command of Twice. This machine is over-actuated (6 motors / 5 dof), but redundancy is helpful to overcome singularities that can appear while inclining the middle part.

\section{Conclusion}

This paper tries to get through one of the drawbacks of PKM: the limitation of tilting angles. An amplification system able to double tilting angles is proposed. After a kinematic description, Twice effectiveness is proved by solving the system leading to internal velocities. Finally some practical improvements are proposed guaranteeing better forces transmission.

\section{Acknowledgements}

This work has been partially supported by the European Commission, in the frame of MACH21 project.

The authors would like to thank Stéphane Guibert, from Observatoire de Haute Provence in France, for his highly valuable remarks regarding this mechanical concept.

\section{References}

[1] J.P. Merlet, Les robots parallèles, Hermes, 1997.

[2] H.K. Tönshoff, "A systematic comparison of parallel kinematics", Keynote in Proc. of the First Forum on PKM, Milan, Italy, August 31-September 1, 1998.

[3] R. Clavel, "Une nouvelle structure de manipulateur parallèle pour la robotique légère", APII, 1989, pp. 501-519.

[4] B. Siciliano, "The Tricept Robot: Inverse kinematics, manipulability analysis and closed-loop direct kinematics algorithm", Robotica, 1999, pp. $437-445$.

[5] C.M. Gosselin, E. St-Pierre and M. Gagné, "On the development of the agile eye: mechanical design, control issues and experimentation", IEEE Robotics and Automation Society Magazine, Vol. 3, No. 4, 1996, pp. 29-37.

[6] S.J. Ryu, J.W. Kim, J.C. Hwang, C. Park, H.S. ho, K. Lee, Y. Lee, U. Cornel, F.C. Park and J. Kim, "ECLIPSE: An Overactuated Parallel Mechanism for Rapid Machining", Proc. ASME Int. Mechanical Engineering Congress and Exposition, U.S.A, 1998, Vol. 8, pp. 681-689.

[7] F. Marquet, S. Krut, O. Company, F. Pierrot, "Archi, a redundant mechanism for machining with unlimited rotation capacities", Proc. of ICAR 2001, Budapest, Aug. 2001.

[8] F. Pierrot, F. Marquet, O. Company and T. Gil, "H4 parallel robot: modeling, design and preliminary experiments", Proc. of IEEE ICR\&A, Seoul, Korea, May 2001.

[9] V.B. Zamanov and Z.M. Sotirov, "Parallel manipulators in robotics", In IMACS/SICE: Int. Symp. on Robotics, Mechatronics, and Manufacturing Systems, Kobe, 16-20 Sept. 1992, pp. 409-418. 